\section{SARCOMA DEL ESTROMA PROSTÁTICO EN UN PACIENTE DE 20 AÑOS DE EDAD.}

\author{
Concepción Lara ${ }^{1}$, Juan José Borrero ${ }^{1}$, Virginia Porras ${ }^{1}$ \\ y J. G iraldez².
}

Servicio de Anatomía Patológica y Urología ${ }^{2}$. Hospital Juan Ramón Jiménez. Huelva. España.

\begin{abstract}
Resumen.- O BJETIVO S: Los tumores de prósta ta representan la neoplasia maligna más frecuente en el varón. M ientras que las lesiones epiteliales son frecuentes, las neoplasias de origen mesenquimal representan solo un 0 ' $2 \%$ de las neoplasias malignas prostáticas y son poco conocidas, sobre todo las de origen estromal.
\end{abstract}

M ETO DO S Y RESULTADO S: Este artículo describe un caso de un varón de 20 años de edad, con un sarcoma del estroma prostático al que se le realiza una cistoprostatectomía radical. El tumor ocupa prácticamente la totalidad de la próstata, alcanzando un diámetro de $8 \mathrm{~cm}$ Histológicamente está constituido por células fusiformes con moderada atipia y frecuentes figuras de mitosis, entremezcladas con aisladas estructuras ductales elongadas, adoptando un patrón similar al tumor filodes, que plantea el diagnóstico diferencial con otros tumores mesenquimales prostáticos.
CON CLUSIONES: El comportamiento clínico de estos tumores, basado en los escasos casos descritos en la literatura, es prácticamente desconocido. Las recurrencias locales no son raras, y se han descrito casos de metástasis a distancia, fundamentalmente a pulmón y hueso. En nuestro caso el paciente permanece libre de enfermedad a los 12 meses del diagnóstico.

Palabras clave: Sarcoma. Estroma prostático.

Próstata.

Summary- O BJEC TIVE: Prostatic tumors are the most frequent malignant neoplasms in men, most of them being constituted by carcinomas; only $0,2 \%$ of malignant prostatic neoplasms are of mesenchimal origin. They are not well known, especially those of prostatic stromal phenotype.

METHODS AN D RESULTS: We report the case of a 20 year-old man with a prostatic stromal sarcoma. After total cystoprostatectomy a tumor measuring $8 \mathrm{~cm}$ could be seen, replacing almost the whole prostate. Microscopically a spindle cell neoplasia with moderate atypia and a high mitotic index entrapping few elongated prostatic ducts (adopting a phyllodes tumor morphology) was observed.

CON CLUSIO N: The clinical behavior of these infrequent sarcomas is not well-established. Recurrences are not uncommon whereas lung and bone metastases have been described. Twelve months after surgery our patient is alive without evidence of disease.

Keywords: Sarcoma. Prostatic sarcoma. Prostate.

\section{INTRODUCCIÓN}

Los tumores prostáticos de origen mesenquimal representan solo un 0 ' $2 \%$ de las neoplasias malignas prostáticas y son poco conocidas, sobre todo las de origen estromal.

La glándula prostática posee un estroma especializado hormonalmente dependiente, con una compleja interacción estromal-epitelial, que participa en la morfogénesis del epitelio prostático, la diferenciación, proliferación y expresión de proteínas específicas de la próstata (1), postulándose que las lesiones proliferativas del estroma prostático son el resultado de una interacción anómala estromal-epitelial. 
Las lesiones proliferativas del estroma prostático se han clasificado en función de su celularidad, actividad mitótica y presencia de necrosis en tumores del estroma prostático de potencial maligno incierto y sarcomas del estroma prostático (2). Desde el punto de vista morfológico son tumores que pueden adoptar diversos patrones, incluyendo el de tumor filodes, y que típicamente expresan CD34 y receptores de progesterona. Desde el punto de vista histopatológico pueden plantear problemas en el diagnóstico diferencial con lesiones como el rabdomiosarcoma, leiomiosarcoma, carcinosarcoma, y sarcoma fibromixoide de bajo grado.

En cuanto a sus características clínicas, suelen ocurrir en pacientes de edad media y avanzada y los síntomas de presentación más frecuentes son los de retención urinaria, hematuria, dolor rectal o masa palpable abdominal o rectal (1).

Presentamos un caso de sarcoma del estroma prostático en un paciente de 20 años de edad, diagnosticado previamente de rabdomiosarcoma embrionario de vejiga a los 2 años de edad, planteando el diagnóstico diferencial con otros tumores mas frecuentes en este rango de edad y revisión de la literatura.

\section{PRESENTACIÓN DEL CASO}

Varón de 20 años de edad, diagnosticado de rabdomiosarcoma retroperitoneal a los 2 años de edad, realizándose tratamiento quirúrgico con quimioterapia y radiote-

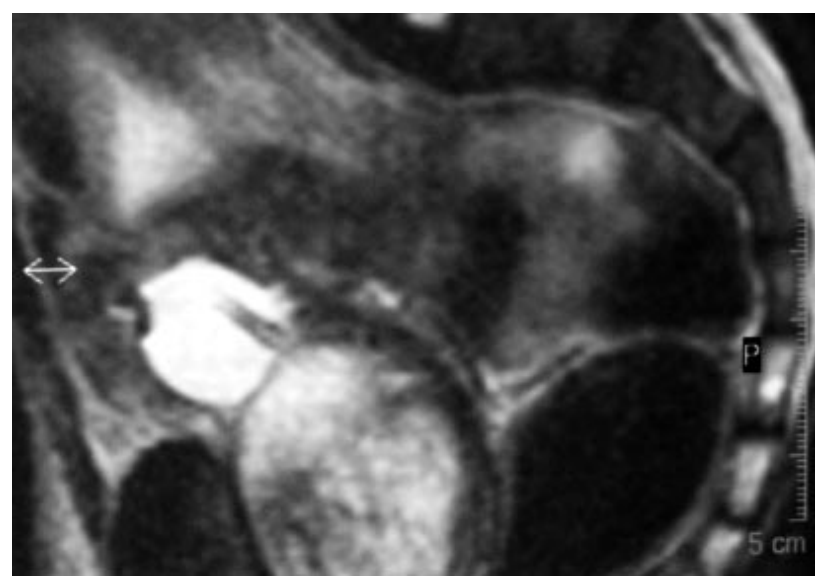

FIG URA 1. RN M. Masa bien delimitada, que parece depender de próstata y desplaza la vejiga. rapia posterior, encontrándose libre de enfermedad neoplásica en los controles posteriores por lo que fue dado de alta cuatro años antes.

A cude a urgencias del Hospital con cuadro de retención urinaria con escapes por rebosamiento y síndrome constitucional con decaimiento y dolor suprapúbico. A la exploración existe debilidad muscular, sensación de ocupación y dolor a la palpación en hipogastrio, donde se aprecia una masa a tensión que se identifica como globo vesical.

En el estudio de imagen (TAC y RNM) se observa una gran ureterohidronefrosis bilateral y una masa bien delimitada, de aspecto encapsulado, que parece depender de la próstata, que infiltra y rechaza hacia delante la microvejiga. No se observaron adenopatías. (Figura 1).

Se practica punción-biopsia transrectal ecodirigida de próstata bajo anestesia general, y con el diagnóstico de sarcoma mixoide se practica cistoprostatectomía radical con derivación de ambos uréteres en cañón de escopeta. En el estudio de extensión no se observaron metástasis y no se realizó tratamiento posterior adyugante.

En el estudio macroscópico de la pieza, se observa una tumoración de $8 \mathrm{~cm}$ de diámetro máximo ocupando la próstata y englobando las vesículas seminales, no reconociéndose parénquima prostático conservado.

En el estudio microscópico se observa un tumor constituido por células fusiformes, con leve atipia citológica y fre-

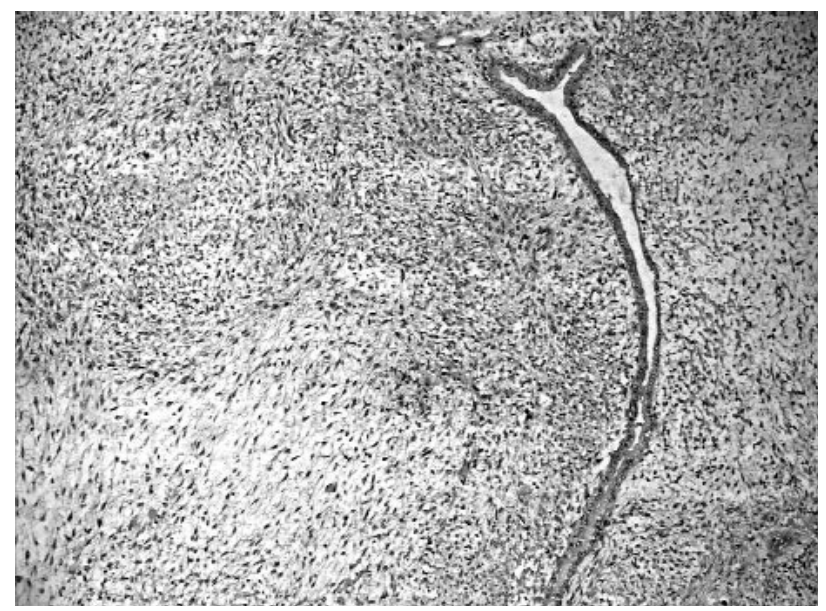

FIG URA 2. Sarcoma del estroma prostático. Area de hipercelularidad estromal, con estructuras glandulares benignas elongadas. Hema toxilina eosina $10 \mathrm{X}$. 
cuentes figuras de mitosis, dispuestas con un patrón laxo, con áreas de mayor densidad celular. Inmersas en el tumor se ven estructuras glandulares elongadas, correpondientes a estructuras glandulares prostáticas no neoplásicas (Figura 2). Se realizó estudio inmunohistoquímico para determinación de CD34, Vimentina, citoqueratina de amplio espectro, Actina músculo liso específica, Desmina, proteína S-100, CD117, Ki67, PSA y receptores de estrógenos y progesterona (Dako Cytomation, Carpinteria, California), observándose expresión generalizada de CD34 y vimentina, con expresión aislada de receptores de progesterona y ausencia del resto de marcadores.

\section{DISCUSIÓN}

Los tumores del estroma prostático de potencial maligno incierto y los sarcomas del estroma prostático son lesiones raras, caracterizadas por expansión del estroma especializado de la próstata, asociado a componente glandular no neoplásico, que típicamente expresan CD34 y receptores de progesterona, esta última característica apoya la teoría de que estas lesiones son el resultado de una respuesta hormonodependiente anómala del estroma prostático especializado.

Muchos autores han asociado este tipo de lesiones a neoplasia mixtas, epiteliales-mesenquimales, que ocurren en otros órganos como el tumor filodes (3).

Desde el punto de vista anatomopatológico, se plantea el diagnóstico diferencial con neoplasias como el rabdomiosarcoma, leiomiosarcoma, carcinosarcoma y el sarcoma fibromixoide de bajo grado. El rabdomiosarcoma es un tumor que ocurre en la infancia, primera década de la vida, y típicamente no expresa CD34 ni progesterona $(4,5)$.

El leiomiosarcoma suele expresar en el estudio inmunohistoquímico actina y muchos de ellos desmina. El carcinosarcoma es un tumor bifásico, compuesto por elementos malignos epiteliales y mesenquimales. El sarcoma fibromixoide de bajo grado no presenta componente epitelial benigno asociado, ni expresión, o solo focal, de CD34 $(1,2)$.

En cuanto a sus características clínicas, suelen darse en un rango de edad entre la tercera y novena déca$d a$, con un pico de incidencia en la sexta y séptima década. N uestro caso presenta la peculiaridad de la temprana edad de presentación y el antecedente de irradiación tras el diagnóstico de rabdomiosarcoma 18 años antes. La asociación de la neoplasia actual con la irradiación previa es incierta, aunque existen casos descritos en la literatura de neoplasias prostáticas, sarcomas, desarrolladas tras radioterapia en el tratamiento de cáncer prostático, con periodos de latencia similares al descrito $(6,7)$. Los síntomas de presentación más frecuentes suelen ser: retención urinaria y hematuria, en un tercio de los pacientes, y menos frecuente dolor rectal o masa palpable abdominal o rectal.

El comportamiento clínico de estos tumores es prácticamente desconocido, dada su escasa frecuencia. Las recurrencias locales no son raras, y se han descrito casos de metástasis a distancia, fundamentalmente a pulmón y hueso (1). En nuestro caso el paciente permanece libre de enfermedad a los 12 meses del diagnóstico.

\section{BIBUOGRAFÍA y LECTURAS RECOMENDADAS (*lectura de interés y **lectura fundamental)}

**1. GUADIN, P.B.; ROSAI, J.; EPSTEIN, J.I.: "Sarcomas and related proliferative lesions of specialized prostatic stroma: a clinicopathologic study of 22 cases". Am. J. Surg. Pathol., 22: 148, 1998.

**2. NEBLE,J.; SAQUTER, G.; EPSTEIN, J.I. y cols.: "Tumours of the urinary system and male genital organs". Capítulo 3. Tumours of the prostate. pp. 209211. IARC Press: Lyon 2004.

3. PROBERT, J.L.; O’ROURKE, J.S.; FARROW, R. y cols.: "Stromal sarcoma of the prostate". Eur. J. Surg. Oncol., 26: 100, 2000.

4. OSAKI, M.; TAKAHASHI, C.; MIYAGAWA, T. y cols.: "Prostatic stromal sarcoma: case report and review of the literature". Pathol. Int., 53: 407, 2003.

*5. YOUNG, R.H.; SRIGLEY, R.J.; AMIN, M.B. y cols.: "Tumors of the prostate gland, seminal vesicles, male urethra, and penis". Capítulo 6. Miscellaneous tumors of the prostate. pp. 270-274. AFIP. Washington, D.C. 2000.

6. AUDET, J.F.; RUIZ, L.; SEBE ,P. y cols.: "Neoplasms induced by radiotherapy for prostate cancer: report of a case of pelvic sarcoma and review of the literature". Prog. Urol., 14: 420, 2004.

7. CANCIELD, S.E.; GANS, T.H.; UNGER, P. y cols.: "Postradiation prostatic sarcoma: de novo carcinogenesis or dedifferentiation of prostatic adenocarcinoma”. Tech. Urol., 7: 294, 2001. 